\title{
An Improved Indoor Positioning Technique Based on Receiving Signal's Strength
}

\author{
Xingsi Xue $\mathbb{D}^{1,},{ }^{1,2,3,4,5,6}$ Xiaoquan Lin, ${ }^{6,7}$ Chaofan Yang, ${ }^{6}$ and Xiaojing Wu ${ }^{6}$ \\ ${ }^{1}$ Fujian Provincial Key Laboratory of Big Data Mining and Applications, Fujian University of Technology, Fuzhou 350118, China \\ ${ }^{2}$ Guangxi Key Laboratory of Automatic Detecting Technology and Instruments, Guilin University of Electronic Technology, \\ Guilin 541004, China \\ ${ }^{3}$ Intelligent Information Processing Research Center, Fujian University of Technology, Fuzhou 350118, China \\ ${ }^{4}$ Fujian Key Lab for Automotive Electronics and Electric Drive, Fujian University of Technology, Fuzhou 350118, China \\ ${ }^{5}$ Institute of Artificial Intelligence, Fujian University of Technology, Fuzhou 350118, China \\ ${ }^{6}$ College of Information Science and Engineering, Fujian University of Technology, Fuzhou 350118, China \\ ${ }^{7}$ Anktion (Fujian) Technology Company Limited, Fuzhou 350003, China
}

Correspondence should be addressed to Xingsi Xue; jack8375@gmail.com

Received 26 June 2020; Revised 14 July 2020; Accepted 22 July 2020; Published 11 August 2020

Academic Editor: Chi-Hua Chen

Copyright (c) 2020 Xingsi Xue et al. This is an open access article distributed under the Creative Commons Attribution License, which permits unrestricted use, distribution, and reproduction in any medium, provided the original work is properly cited.

\begin{abstract}
Wireless signal-transmitting process is a complex procedure, to improve the indoor positioning accuracy, and this work proposes a novel indoor positioning technique based on receiving signal's strength. First, the indoor environment of the building is regionalized in the training phase of indoor positioning. Then, the adjacent points of the indoor space with the same wireless signal transmission characteristics are gathered into the same area, and the corresponding parameter sets and decision domains of each area are constructed. After that, during the positioning stage, the regional confidence and receiving signal's strength are used to predict the indoor area where the mobile station is located. Finally, the ranging and solution results of the traditional three-sided positioning process are constrained to obtain the optimal solution. Comparing with the traditional positioning techniques that regard the entire complex indoor environment as an entirety, the proposed indoor space regionalization preprocessing method can effectively reduce the ranging error. Compared with the indiscriminate data fusion of the centroid method, the data filtering method based on regional confidence is more targeted. In the experiment, a practical office area is used to test our proposal's performance, and the experimental results show that our approach can effectively improve the accuracy of indoor positioning results.
\end{abstract}

\section{Introduction}

With the advancement of information technology and the development of society, Location-Based Service (LBS) has become a basic service requirement for people's daily work and life [1]. The positioning technique can be divided into two types: outdoor positioning technique and indoor positioning technique. In an outdoor environment, Global Positioning System (GPS), BeiDou Navigation Satellite System (BDS), and other Global Navigation Satellite System (GNSS) provide users with meter-level location services, solved the problem of accurate positioning in outdoor space basically [2]. However, in an indoor environment that occupies $80 \%$ of human daily life, the GNSS signal strength is drastically reduced due to the blocking effect of buildings on wireless signals. As a result, the positioning accuracy is greatly reduced and cannot meet the needs of indoor location services. This problem is particularly prominent in large shopping malls, integrated transportation hubs, underground mines, etc., in large and complex indoor environments. Therefore, how to improve the accuracy of indoor positioning technique in a large and complex indoor environment is the current research focus in the field of positioning technique [3]. Indoor positioning technique based 
on receiving signal's strength (RSS) has become the mainstream indoor positioning technique because it has the characteristics that it can directly use the existing widely deployed WLAN equipment and the low cost of hardware is easy to deploy.

The indoor positioning technique based on RSS can be mainly divided into the positioning technique based on ranging and the positioning technique based on location fingerprint [4]. The location method based on location fingerprint is to detect the wireless signal characteristics of a specific location and build a location fingerprint database based on this. During the positioning stage, the wireless signal characteristics are detected and a specific matching algorithm is used to traverse the location fingerprint database through calculation to estimate the location information of the target node. The location fingerprint positioning method does not require the specific information of the reference point and has unique advantages in specific occasions where it is impossible or inconvenient to calibrate the reference point. However, the location fingerprint positioning method requires considerable workload to establish and maintain the location fingerprint database. The range-based positioning method uses the attenuation characteristics of the wireless signal during transmission to determine the distance between the target node and multiple reference points whose positions are known. On this basis, the location information of the target node is obtained by further calculation. The positioning method based on distance measurement is easy to deploy, low in cost, low in maintenance, and easy to promote. However, due to the complexity and diversity of the propagation path of wireless signals in an indoor environment, a single wireless signal transmission model cannot describe the propagation characteristics of wireless signals at different points indoors, resulting in large errors and lower positioning accuracy. In order to accurately determine the area of the point to be measured and further improve the positioning accuracy, this paper designs and implements a judgment method based on regional confidence. This method uses the error vector of the equation and the degree of compatibility to filter out the irrelevant combination of regions; thus, making the determination position of the point to be measured is more accurate.

\section{Indoor Positioning Technique}

With the vigorous development of information technology and electronic technology, indoor positioning methods continue to emerge and have been applied. The widely used indoor positioning methods include proximity detection, triangulation, polar point method, dead reckoning, finger printing, and multilateration [5]. The proximity detection method is to detect the coverage area of the launch point where the target is located to determine the approximate range where the target is located, with low accuracy. The triangular positioning method and pole method mainly determine the coordinates of the target by observing the angle relationship between the target and the known reference point. Dead reckoning is positioning by acquiring the movement speed, direction, and time of the measured target. It is convenient to use but has accumulated errors. When it is used in an indoor environment, it has a pedestrian dead reckoning (PDR) algorithm for pedestrian positioning scenarios. Fingerprint positioning is a method of establishing a fingerprint map database in advance and matching the collected signal fingerprint data with database data records during positioning to determine the target location. Fingerprint positioning does not require reference point position information and is very suitable for special occasions where reference point position information cannot be calibrated. However, the workload of establishing and maintaining the fingerprint database is very large, and achieving efficient data matching has always been a difficult problem, which has attracted the attention of many scholars. Some scholars have made other progress in the field of ontology research and made some progress [6-15]. The multilateral positioning method is to calibrate the positions of several reference points in advance and then determine the position of the point to be measured by measuring the distance between the point to be measured and the reference point. In practical applications, three reference points are generally used for positioning, which is also called three-sided positioning.

Different positioning methods need to choose different observations. Common observations include Time Of Arrival (TOA), Time Difference Of Arrival (TDOA), Enhanced Observed Time Difference (EOTD), and Round Trip Time (RTT), Arrival Of Angle (AOA), receiving signal's strength (RSS), etc. The fingerprint positioning method, the trilateral positioning method, and the multilateral positioning method usually use RSS as the observation method for positioning.

Based on these indoor positioning methods, various indoor positioning technologies have been derived and developed [16], which are compared in Table 1.

The technologies for trilateral positioning based on RSS mainly include Wifi, Bluetooth, RFID, ZigBee, and so on. Because of its convenient data collection, a small amount of calculation, and extensive network deployment, it has a good application prospect.

\section{Wireless Signal Transmission Model and Trilateral Positioning Algorithm}

Calculating the distance between the point to be measured and the reference point using the distance measurement method and then using the trilateration algorithm to calculate the position of the point to be measured is a typical method of RSS-based positioning technique, which is simple to calculate and has good universality.

3.1. Wireless Signal Transmission Model. The wireless signal transmission model is a mathematical model in which wireless signals propagate through a medium in a certain medium environment. Through the wireless signal transmission model, a quantitative relationship between the degree of signal attenuation caused by the transmission process of the wireless signal in the medium space and the transmission distance can be obtained, and a reasonable selection of the signal transmission model and transmission 
TABLE 1: Comparison of main indoor positioning technologies.

\begin{tabular}{|c|c|c|c|c|}
\hline $\begin{array}{l}\text { Positioning } \\
\text { technique }\end{array}$ & Positioning method & $\begin{array}{c}\text { Positioning } \\
\text { accuracy }\end{array}$ & Advantages & Disadvantages \\
\hline Visual image & $\begin{array}{c}\text { Image processing, scene } \\
\text { analysis }\end{array}$ & High & $\begin{array}{c}\text { Low environmental } \\
\text { dependence }\end{array}$ & High cost and low stability \\
\hline Infrared & $\begin{array}{l}\text { Image processing, proximity } \\
\text { detection }\end{array}$ & High & Mature technology & $\begin{array}{l}\text { Visual distance transmission, } \\
\text { interfered by light }\end{array}$ \\
\hline Ultrasound & Multilateral positioning & High & High positioning accuracy & $\begin{array}{c}\text { Greatly affected by temperature } \\
\text { and loss }\end{array}$ \\
\hline Bluetooth & $\begin{array}{c}\text { Proximity detection, } \\
\text { multilateral positioning }\end{array}$ & Medium & $\begin{array}{l}\text { Small size and low power } \\
\text { consumption }\end{array}$ & $\begin{array}{c}\text { Short transmission distance and } \\
\text { low stability }\end{array}$ \\
\hline Wifi & $\begin{array}{l}\text { Fingerprint positioning, } \\
\text { multilateral positioning }\end{array}$ & Medium & Low cost and easy deployment & $\begin{array}{l}\text { Susceptible to environmental } \\
\text { interference }\end{array}$ \\
\hline RFID & $\begin{array}{l}\text { Proximity detection, } \\
\text { fingerprint positioning }\end{array}$ & High & Low cost and small size & $\begin{array}{l}\text { Short distance and weak } \\
\text { communication ability }\end{array}$ \\
\hline Ultrawideband & Multilateral positioning & High & Strong penetration & High cost \\
\hline Geomagnetic & Fingerprint positioning & Medium & $\begin{array}{c}\text { Not dependent on the } \\
\text { environment }\end{array}$ & Poor stability \\
\hline $\begin{array}{l}\text { Cellular base } \\
\text { station }\end{array}$ & Proximity detection & Low & Wide coverage & Depends on base station density \\
\hline ZigBee & $\begin{array}{l}\text { Proximity detection, } \\
\text { multilateral positioning }\end{array}$ & High & $\begin{array}{l}\text { Low cost and low power } \\
\text { consumption }\end{array}$ & $\begin{array}{c}\text { Poor stability and susceptible to } \\
\text { interference }\end{array}$ \\
\hline Pseudolite & Carrier phase ranging & High & Wide coverage & High cost \\
\hline Inertial navigation & Dead reckoning & Medium & $\begin{array}{c}\text { Not dependent on the external } \\
\text { environment }\end{array}$ & Cumulative error \\
\hline
\end{tabular}

model parameters suitable for the current environment is satisfactory indoor positioning performance is crucial. The propagation model of wireless signals in free space suggests that the receiving signal's strength $P_{r}$ can be expressed by the following formula [16]:

$$
P_{r}(d)=\frac{P_{t} G_{t} G_{r} \lambda^{2}}{(4 \pi)^{2} d^{2} L},
$$

where $P_{t}$ is the signal power at the transmitter, $\lambda$ is the wavelength of the wireless signal, $G_{t}$ is the antenna gain at the transmitter, $G_{t}$ is the antenna gain at the receiver, $d$ is the separation distance between the transmitter and receiver, and $L$ is the system loss factor. It is troublesome to directly use the free space propagation model for ranging and positioning, so the logarithmic distance loss model is commonly used for indoor positioning [17]:

$$
P(d)=P\left(d_{0}\right)-10 n \lg \left(\frac{d}{d_{0}}\right)+X .
$$

The above formula $P(d)$ is the signal strength received by the receiving end whose distance source (transmitter) is $d$, that is, the RSS value. $P\left(d_{0}\right)$ is the signal strength received by the receiver when the distance from the source is $d_{0} . n$ is the path loss index, which is usually obtained by actual measurement. Generally, the more the obstacles on the propagation path, the larger the value of $n$, so that the wireless signal decreases more in the unit propagation distance [18]. $d_{0}$ is the reference distance, which depends on the actual situation on-site. For the convenience of calculation and measurement, the value is usually $1 . X$ is a Gaussian random variable in $\mathrm{dBm}$ with a mean value of 0 and a variance ranging from 4 to 10 [19].

In engineering applications, the following simplified form of the logarithmic distance loss model is commonly used [20]:

$$
R=A-10 n \lg (d)
$$

The argument $d$ is the distance between the source and the receiver, $R$ is the signal strength received by the receiver, $A$ is the signal strength received $1 \mathrm{~m}$ from the source, and $n$ is the path loss. During application, it is necessary to select several sample points in different positions in advance, then determine $A$ and $n$ parameters of the model through regression analysis, and thus establish the quantitative relationship between RSS and $d$ detected by the receiving end. In the positioning stage, the distance $d$ between the point to be measured and the source can be determined based on the RSS value received by the point to be measured.

3.2. Trilateral Positioning Algorithm. The currently widely used positioning method is the trilateral positioning algorithm [21]. On a two-dimensional plane, if the distance between the point to be measured and three reference points (i.e., sources) with known positions can be determined, the three reference points are used as the center of the circle to measure the distance between the source and the reference point. A circle is made for the radius. Figure 1 shows an example of three-sided positioning technique.

In the three-dimensional space, four reference points are needed for spherical intersection for positioning calculation. 


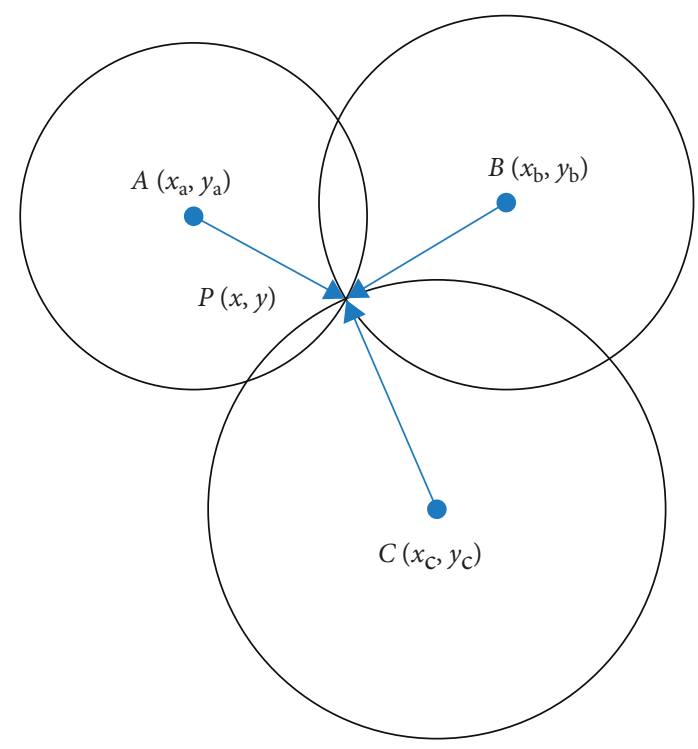

Figure 1: An example of three-sided positioning technique.

Traditional RSS indoor positioning technique considers the entire indoor environment as a whole during the entire positioning process and only uses a single wireless signal transmission model and corresponding model parameters for distance calculation. In complex indoors with many barriers, the environment will inevitably produce a large error, and due to the influence of the ranging error, the three-sided positioning algorithm is likely to not intersect the three circles at a point, but to a common area. In extreme cases, they are also may be completely disjointed, resulting in a large error in the calculation results of positioning. Even if some methods such as the centroid method are used to modify and approximate the position, the results are not satisfactory. This paper improves this and proposes a new method, which uses the indoor space regional preprocessing method and regional confidence discrimination method to solve the above problems.

\section{Improved RSS Indoor Positioning Technique}

The main reason for the low accuracy of the traditional RSS indoor positioning method based on three-sided positioning is that the indoor environment is complicated. The situation of the obstructions on the propagation path of the wireless signal from the source to the indoor points is different. In the wireless signal strength of each propagation path, the decay rate is very different. The single set of propagation model parameters used in this method ignores these differences and naturally introduces large errors. To this end, this paper first conducts regionalization processing and divides the indoor environment into several areas according to the wireless signal transmission characteristics. Each point in the same area selects the same transmission model parameters, and different areas correspond to different transmission model parameters. In order to simplify the calculation, the transmission model of each region is set as the logarithmic distance loss model. In the positioning stage, the region where the point to be measured is located is determined by the region confidence judgment method, and the model parameters corresponding to the region are selected for accurate positioning calculation. Since the difference in the wireless signal transmission path from each point in the room to the source under the spatial layout of the indoor environment is fully considered, the positioning accuracy can be greatly improved.

4.1. Regionalization of Interior Space. The path loss factor $n$ of the logarithmic distance loss model characterizes the attenuation rate of wireless signals on the unit transmission path and fixed hard barriers such as walls affect the value of the parameter $A$. According to this, the indoor space can be divided into several areas according to the number and size of obstructions on the wireless signal transmission path.

As shown in Figure 2, in a specific indoor space, three sources of $A P 1, A P 2$, and $A P 3$ are deployed. There are two walls in the room, namely, wall $f h$ and wall $e k$. To simplify the analysis and highlight the problem elements, assume the wall the thickness is 0 . It can be seen from the figure that the wireless signal sent from the source $A P 1$ passes through two representative paths, namely, path $a j$ and path ai. Obviously, the difference between the two paths is significant, the data of the barriers (walls) on each path are different, and the wireless signal transmission characteristics on each path are also different; that is, the corresponding parameters in each path change accordingly. According to the obstruction on the wireless signal transmission path, the indoor space can be divided into regions according to the source $A P 1$, as shown in Figure 3.

As can be seen from Figure 3, the indoor space is divided into three areas as $z_{1,1}, z_{1,2}$, and $z_{1,3}$ according to the source $A P 1$, and the parameter values of the logarithmic distance loss model corresponding to each area (that is, the values of $A$ and $n$ ) are different and determined by the actual situation. These areas constitute the indoor space that is divided into regions based on the source $A P 1$ and $Z_{1}$ are recorded as their union, which is defined as follows:

$$
Z_{1}=z_{1,1} \cup z_{1,2} \cup z_{1,3}=\left\{z_{1,1}, z_{1,2}, z_{1,3}\right\} \text {. }
$$

Obviously, it is known that $Z_{1}$ and the actual size of the indoor space are in one-to-one correspondence and completely coincide. This article defines it as the plane corresponding to the source $A P 1$. As can be seen from the foregoing, the parameters of the wireless signal transmission model corresponding to each area included in the plane $Z_{1}$ are generally different, and $c_{1,1}=\left\{\widehat{A_{11}}, t \widehat{n_{11}}\right\}$ is a set of transmission parameters corresponding to the area $z_{1,1}$. Among them, $\widehat{A_{11}}$ and $\widehat{n_{11}}$ are the estimated values of parameters $A$ and $n$ in the area $z_{1,1}$ in formula (3). Similarly, it can be constructed as $c_{1,1}, c_{1,2}$, and $c_{1,3}$. Let $C_{1}$ be the parameter set corresponding to the plane $Z_{1}$, then $C_{1}=c_{1,1} \cup c_{1,2} \cup c_{1,3}=\left\{c_{1,1}, c_{1,2}, c_{1,3}\right\}$. Similarly, based on the source $A P 2$ and source $A P 3$, the plane $Z_{2}$ and plane $Z_{3}$ and corresponding parameter sets $C_{2}$ and $C_{3}$ can be constructed. As can be seen from Figure $3, z_{1,1}$ is a polygon composed of multiple line segments. In general, the region $z_{1,1}$ is defined as follows: 


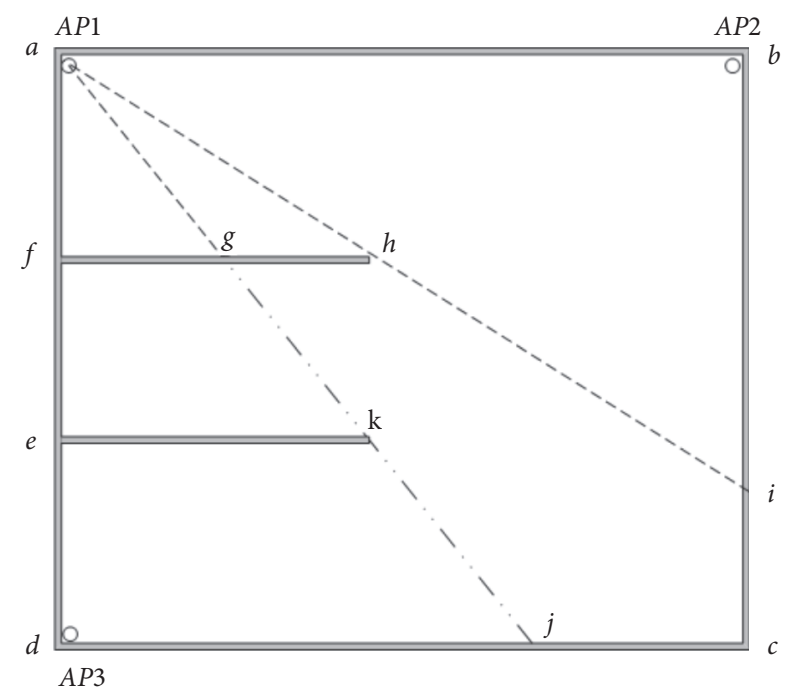

Figure 2: Schematic diagram of obstacles in the wireless signal transmission path of the indoor environment.

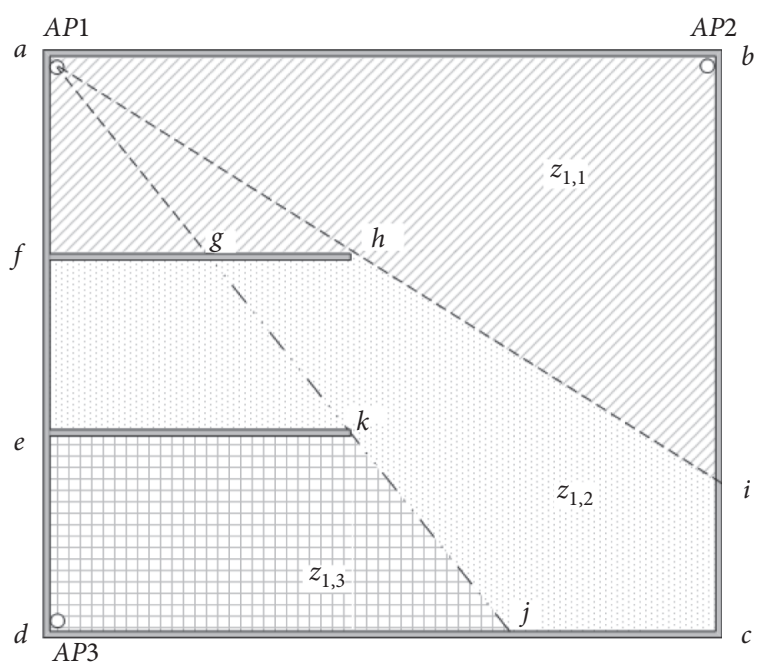

Figure 3: Schematic diagram of the regional division of indoor environmental space.

$$
z_{1,1}=\left\{(x, y) \mid f_{i}(x, y)<0, \quad i=1,2, \ldots, r\right\},
$$

where $f_{i}(x, y)$ is an analytical formula of straight lines that constitute the outline of a polygon and $r$ is 5 in the area $z_{1,1}$.

$\left\|p_{1}-p_{2}\right\|$ is recorded as the normal distance between points $p_{1}$ and $p_{2}$, the point $p(x, y)$ is any point on $z_{1,1}$, and the point $a\left(x_{a}, y_{a}\right)$ is the location of a source $A P 1$. Then, from equations (3) and (5), the RSS minimum value $R_{a 1}$ based on the source AP1 that can be obtained by all points located in the area $z_{1,1}$ is as follows:

$$
\begin{array}{ll}
\min & R_{a 1}=A-10 n \lg \left(\left\|p(x, y)-a\left(x_{a}, x y_{a}\right)\right\|\right), \\
\text { s.t. } & f_{i}(x, y)<0, \quad i=1,2, \ldots, r .
\end{array}
$$

Obviously, equation (7) is a linear programming problem, and the minimum value of $R_{a 1}$ can be solved by the simplex method, which is recorded as $R_{a 1_{-} \text {min }}$. Similarly, the maximum value $R_{a 1_{\text {_max }}}$ of $R_{a 1}$ can be obtained. Supposing the RSS value is calculated based on the source AP1 corresponding to any point $p(x, y)$ on $z_{1,1}$ be $R_{p}$. Considering the connectivity of the area $z_{1,1}$ and the monotonicity of formula (3), there are obviously

$$
\forall p(x, y) \in z_{1,1}, \Longrightarrow R_{p} \in\left[R_{a 1_{-} \min }, R_{a 1_{-} \max }\right] .
$$

In this way, the area $z_{1,1}$ has a corresponding relationship with the $R_{a 1_{-} \min }$ and $R_{a 1_{-} \max }$ values. In this paper, the range determined by the set of values corresponding to the area is defined as the decision area of the area. For example, the above decision area of $z_{1,1}$ is $\left[R_{a 1_{-} \min }, R_{a 1_{-} \max }\right]$, which is denoted as $j_{1,1}$. Similarly, the decision domain $j_{1,2}$ and $j_{1,3}$ corresponding to the remaining regions $z_{1,2}$ and $z_{1,3}$ of the plane $Z_{1}$ can be established. Remember that the set of decision domains in the region contained in the plane $Z_{1}$ is $J_{1}$, and then $J_{1}=\left\{j_{1,1}, j_{1,2}, j_{1,3}\right\}$. With reference to the processing procedure of the plane $Z_{1}$, the same processing can also be performed on the planes $Z_{2}$ and $Z_{3}$ to construct the boundary ranges, parameter sets, and decision domains of the regions in the $Z_{2}$ and $Z_{3}$ planes. So far, the regionalization of indoor space has been completed.

4.2. Positioning. The actual positioning process can be carried out only after the process of indoor space regionalization is completed. For ease of explanation, this article defines the former as the indoor positioning training stage and the latter as the positioning stage.

Assuming that the preprocessing of indoor regionalization has been completed during the training phase, the positions of the three sources $A P 1, A P 2$, and $A P 3$ are determined, and the area where the point $p(x, y)$ to be measured is located is $z_{p}$, and the values of the RSS of the three sources detected at $p(x, y)$ are $R_{1}, R_{2}$, and $R_{3}$, respectively, and the plane $Z$, parameter set $C$, and decision domain set $J$ corresponding to each source are constructed. In the positioning stage, according to different situations in which $R_{1}, R_{2}$, and $R_{3}$ detected at the position of the point $p(x, y)$ to be measured fall within the decision domains corresponding to the respective regions of the planes $Z_{1}, Z_{2}$ and $Z_{3}$ which will be separately explained below.

4.2.1. Ideal Positioning. First, consider the simplest case, that is, $R_{1}, R_{2}$, and $R_{3}$,only falls within the corresponding one decision domain on the three planes of $Z_{1}, Z_{2}$, and $Z_{3}$, namely,

$$
\left\{\begin{array}{l}
R_{1} \in j_{1,1}, \\
R_{2} \in j_{2,1}, \\
R_{3} \in j_{3,1} .
\end{array}\right.
$$

Among them, $j_{1,1}, j_{2,1}$, and $j_{3,1}$ are the corresponding judgment domain in the area $z_{1,1}, z_{2,1}$, and $z_{3,1}$, and it is obvious that 


$$
\left\{\begin{array}{l}
z_{p} \in z_{1,1}, \\
z_{p} \in z_{2,1}, \\
z_{p} \in z_{3,1} .
\end{array}\right.
$$

Therefore, the transmission parameters relative to the three sources can be obtained by $z_{p}$ as $c_{1,1}, c_{1,2}$, and $c_{1,3}$. From equation (3), combined with known conditions, the following equations can be obtained:

$$
\left\{\begin{array}{l}
\left(x-x_{1}\right)^{2}+\left(y-y_{1}\right)^{2}=d_{1}^{2} \\
\left(x-x_{2}\right)^{2}+\left(y-y_{2}\right)^{2}=d_{2}^{2} \\
\cdots \\
\left(x-x_{n}\right)^{2}+\left(y-y_{n}\right)^{2}=d_{n}^{2} .
\end{array}\right.
$$

This is the system of equations about $x$ and $y$, where $x$ and $y$ are the position coordinate of the point $p(x, y)$ to be measured, and $\left(x_{1}, y_{1}\right),\left(x_{2}, y_{2}\right)$, and $\left(x_{3}, y_{3}\right)$ are the position coordinates of the source $A P 1$, source $A P 2$, and source $A P 3$, respectively, which are known constants. $d_{1}, d_{2}$, and $d_{n}$ are the distances between the point to be measured $p(x, y)$ and each source, which is calculated by using the transmission parameters $c_{1,1}, c_{2,1}$, and $c_{3,1}$ of $z_{p}$ relative to each source and combining formula (3). According to the title, there are only 3 sources deployed in the indoor environment, so the value of $n$ in equation (11) is 3 .

Execute the linear transformation on equation (11) with $r_{(k+1) k}(-1), k=1,2, \ldots, n-1$ eliminate the quadratic term and sort out, and then the result can be expressed as follows:

$$
\mathbf{G W}=\mathbf{b} .
$$

Here, G, W, and $\mathbf{b}$ are defined as follows:

$$
\begin{aligned}
\mathbf{G} & =\left[\begin{array}{cc}
2\left(x_{2}-x_{1}\right) & 2\left(y_{2}-y_{1}\right) \\
2\left(x_{3}-x_{2}\right) & 2\left(y_{3}-y_{2}\right) \\
\vdots & \vdots \\
2\left(x_{n}-x_{n-1}\right) & 2\left(y_{n}-y_{n-1}\right)
\end{array}\right], \\
\mathbf{W} & =\left[\begin{array}{c}
x \\
y
\end{array}\right], \\
\mathbf{b} & =\left[\begin{array}{c}
d_{1}^{2}-d_{2}^{2}-x_{1}^{2}+x_{2}^{2}-y_{1}^{2}+y_{2}^{2} \\
d_{2}^{2}-d_{3}^{2}-x_{2}^{2}+x_{3}^{2}-y_{2}^{2}+y_{3}^{2} \\
\vdots \\
d_{n-1}^{2}-d_{n}^{2}-x_{n-1}^{2}+x_{n}^{2}-y_{n-1}^{2}+y_{n}^{2}
\end{array}\right],
\end{aligned}
$$

where $\mathbf{W}$ is the position of the point to be measured that is expected to be obtained. When the number of sources is greater than $3(n>3)$, the form of equation (11) remains unchanged. Due to hardware limitations and measurement accuracy, if equation (11) is used to solve the position of the point $p(x, y)$ to be measured in engineering applications, there may be no solution. The following uses the maximum likelihood method to convert equation (11).
Let $g_{i j}, w_{j}$, and $b_{i}$ be the elements of the matrix, respectively, where $i=1,2, \ldots, n$ and $j=1,2$, then equation (11) can be expressed as

$$
g_{i 1} w_{1}+g_{i 2} w_{2}=b_{i}, \quad i=1,2, \ldots, n .
$$

Since the measurement of the RSS values of each source at the point $p(x, y)$ to be measured is independent of each other,

$$
b_{i}=g_{i 1} w_{1}+g_{i 2} w_{2}+\varepsilon_{i}, \quad \varepsilon_{i} \sim N\left(0, \sigma^{2}\right), i=1,2, \ldots, n .
$$

Then, we get

$$
b_{i} \sim N\left(g_{i 1} w_{1}+g_{i 2} w_{2}, \sigma^{2}\right), \quad i=1,2, \ldots, n .
$$

Finally, the joint density of $b_{i}$ is

$$
\begin{aligned}
\mathbf{L}_{\mathbf{w}} & =\prod_{i=1}^{n} \frac{1}{\sigma \sqrt{2 \pi}} \exp \left[-\frac{1}{2 \sigma^{2}}\left(b_{i}-g_{i 1} w_{1}-g_{i 2} w_{2}\right)^{2}\right] \\
& =\left(\frac{1}{\sigma \sqrt{2 \pi}}\right)^{n} \exp \left[-\frac{1}{2 \sigma^{2}} \sum_{i=1}^{n}\left(b_{i}-g_{i 1} w_{1}-g_{i 2} w_{2}\right)^{2}\right] .
\end{aligned}
$$

When the maximum value of $\mathbf{L}_{\mathbf{w}}$ is obtained, the corresponding is the estimated position value of the point to be measured, and set

$$
\mathbf{Q}_{\mathbf{w}}=\sum_{i=1}^{n}\left(b_{i}-g_{i 1} w_{1}-g_{i 2} w_{2}\right)^{2} .
$$

Taking the logarithm of both sides of $\mathbf{L}_{\mathbf{w}}$ and taking into account the sign and constant terms, it is obvious that the maximum value obtained by $\mathbf{L}_{\mathrm{w}}$ is equivalent to the minimum value obtained by $\mathbf{Q}_{\mathbf{w}}$. For the convenience of subsequent calculations, transform $\mathbf{Q}_{\mathbf{W}}$ into matrix form:

$$
\mathbf{Q}_{\mathbf{W}}=(\mathbf{b}-\mathbf{G W})^{\mathrm{T}}(\mathbf{b}-\mathbf{G W}) \text {. }
$$
get

Expand and organize the right term of formula (18) to

$$
Q_{W}=\mathbf{b}^{\mathrm{T}} \mathbf{b}-2 \mathbf{W}^{\mathrm{T}} \mathbf{G}^{\mathrm{T}} \mathbf{b}+\mathbf{W}^{\mathrm{T}} \mathbf{G}^{\mathrm{T}} \mathbf{G W} .
$$

Then, derivate the two sides of equation (19):

$$
\begin{aligned}
\frac{\partial \mathbf{Q}_{\mathbf{W}}}{\partial \mathbf{W}} & =\frac{\partial\left(\mathbf{b}^{\mathrm{T}} \mathbf{b}-\mathbf{2} \mathbf{W}^{\mathrm{T}} \mathbf{G}^{\mathrm{T}} \mathbf{b}+\mathbf{W}^{\mathrm{T}} \mathbf{G}^{\mathrm{T}} \mathbf{G W}\right)}{\partial \mathbf{W}} \\
& =-2 \mathbf{G}^{\mathrm{T}} \mathbf{b}+\mathbf{2} \mathbf{G}^{\mathrm{T}} \mathbf{G W} .
\end{aligned}
$$

Let equation (20) be 0 , and after shifting the terms, we get

$$
\mathbf{G}^{\mathrm{T}} \mathbf{G W}=\mathbf{G}^{\mathrm{T}} \mathbf{b} \text {. }
$$

Equation (21) is the result of equation (11) converted by the maximum likelihood method.

From equation (21), Let $r$ be the rank of the matrix G, namely, 


$$
r=\operatorname{rank}(\mathbf{G})
$$

Then, there must be

$$
\operatorname{rank}\left(\mathbf{G}^{\mathrm{T}} \mathbf{G}\right)=r .
$$

At the same time, the augmented matrix of equation (21) can be expressed as

$$
\left(\mathbf{G}^{\mathrm{T}} \mathbf{G}, \mathbf{G}^{\mathrm{T}} \mathbf{b}\right)=\mathbf{G}^{\mathrm{T}}(\mathbf{G}, \mathbf{b})
$$

because

$$
\operatorname{rank}\left(\mathbf{G}^{\mathbf{T}}(\mathbf{G}, \mathbf{b})\right) \leq \operatorname{rank}\left(\mathbf{G}^{\mathbf{T}}\right)=r .
$$

In addition, $\left(\mathbf{G}^{\mathrm{T}} \mathbf{G}, \mathbf{G}^{\mathrm{T}} \mathbf{b}\right)$ has more columns than $\left(\mathbf{G}^{\mathrm{T}} \mathbf{G}\right)$; obviously,

$$
\operatorname{rank}\left(\mathbf{G}^{\mathrm{T}} \mathbf{G}, \mathbf{G}^{\mathrm{T}} \mathbf{b}\right) \geq \operatorname{rank}\left(\mathbf{G}^{\mathrm{T}} \mathbf{G}\right)=r .
$$

So, there must be

$$
\operatorname{rank}\left(\mathbf{G}^{\mathrm{T}} \mathbf{G}, \mathbf{G}^{\mathrm{T}} \mathbf{b}\right)=\operatorname{rank}\left(\mathbf{G}^{\mathrm{T}} \mathbf{G}\right)=r .
$$

Therefore, it can be seen that equation (21) must not appear without solution. The position of the point to be measured can be obtained by solving $\mathbf{W}$ from equation (21).

4.2.2. General Positioning Processing. If the RSS value of a certain source detected at the position of the point $p(x, y)$ to be measured does not fall into any one of the decision domains of the corresponding decision domain set $J$ through traversal retrieval, this situation indicates that the RSS measurement error is too large, resulting in an error and requires remeasurement, or it may be that the parameter calibration of the transmission model in the training phase has caused an error, and it needs to be carefully checked and re-executed. The following discussion focuses on the more general case; that is, the RSS value falls into multiple decision domains of the corresponding decision domain set of each source.

Assuming that the RSS values $R_{1}, R_{2}$ and $R_{3}$ of the three sources detected by the point $p(x, y)$ to be measured fall within multiple decision domains of the corresponding decision domain set $J$, considering the general situation, there are

$$
\left\{\begin{array}{l}
R_{1} \in\left\{j_{1,1}, j_{1,2}, \ldots, j_{1, m}\right\}, \\
R_{2} \in\left\{j_{2,1}, j_{2,2}, \ldots, j_{2, n}\right\}, \\
R_{3} \in\left\{j_{3,1}, j_{3,2}, \ldots, j_{3, k}\right\} .
\end{array}\right.
$$

According to the correspondence between the source plane $Z$ and the decision domain set $J$, there are

$$
\left\{\begin{array}{c}
z_{p} \in\left\{z_{1,1}, z_{1,2}, \ldots, z_{1, m}\right\}, \\
z_{p} \in\left\{z_{2,1}, z_{2,2}, \ldots, z_{2, n}\right\}, \\
z_{p} \in\left\{z_{3,1}, z_{3,2}, \ldots, z_{3, k}\right\} .
\end{array}\right.
$$

As can be seen from the above formula, the area $z_{p}$ to which the point $p(x, y)$ to be measured belongs is in the plane $Z_{1}, m$ areas meet the conditions, $n$ areas in the plane
$Z_{2}$ meet the conditions, and $k$ areas in the plane $Z_{3}$ meet the conditions. Therefore, the number of equations to be solved is

$$
\left(\begin{array}{c}
m \\
1
\end{array}\right)\left(\begin{array}{l}
n \\
1
\end{array}\right)\left(\begin{array}{l}
k \\
1
\end{array}\right)=m n k .
$$

Obviously, there are $m n k$ solutions for $\mathbf{W}$ according to equation (21). Simply, you can consider using the centroid method to synthesize all the calculation results to estimate the position of the point $p(x, y)$ to be measured:

$$
\widehat{\mathbf{W}}=\frac{1}{m n k} \sum_{i=1}^{m n k} \mathbf{W}_{i},
$$

where $\mathbf{W}_{i}$ is the solution of the $i$-th equation system among the mnk equation systems determined by equation (29). However, considering the known conditions of the problem set, a more precise screening can be made to further improve the positioning accuracy.

According to known conditions, in an ideal situation, equation (11) must be solvable, and $z_{p}$ can only be in one of the regions of $Z_{a}, Z_{b}$, and $Z_{c}$; that is, the situation of equation (9) must occur. However, now $z_{p}$ occupies multiple areas in $Z_{1}, Z_{2}$, and $Z_{3}$. That is, the situation of formula (29) appears, so it is reasonable to believe that due to the influence of a certain factor, the possible solution of formula (11) is deviated, and thus appears the result of equation (29). Examining the various parameters of equation (3) can only be caused by errors introduced by the $R$-value measurement. It can be seen from common sense that the specific position (coordinate value) of the point $p(x, y)$ to be measured has one and only one at a certain time, so the solution of only one equation system among the mnk equation systems determined by equation (30) is the closest to the objective reality. The degree of incompatibility of equation (11) is used as a measure of the degree to which the solution of the equation is close to the objective reality. The equation with the lowest degree of incompatibility is the best choice among $m n k$ equations, and the solution is the optimal solution.

Define the regional confidence $\rho$ as follows:

$$
\rho=1-\frac{c}{\max _{i} c_{i}}, \quad i=1,2, \ldots, m n k, c \in \mathbb{R}, c \geq 0,
$$

where $c$ is a measure of the degree to which the solution of the equation system in the region corresponding to $\rho$ is close to the objective degree and $c_{i}$ is the $c$ value of the equation system in the $i$-th region. Obviously, the value of $\rho$ is between $[0,1]$, and the value of the parameter $c$ is determined below.

According to the above error analysis, the error vector $\mathbf{m}_{\varepsilon}$ is introduced so that the following formula holds:

$$
\mathbf{G W}=\mathbf{b}+\mathbf{m}_{\varepsilon} .
$$

From equation (21), we can see that the estimated value of equation (11) is

$$
\widehat{\mathbf{W}}=\left(\mathbf{G}^{\mathrm{T}} \mathbf{G}\right)^{-1} \mathbf{G}^{\mathrm{T}} \mathbf{b} .
$$

Substitute into (33), and sort out 


$$
\mathbf{m}_{\varepsilon}=\mathbf{G}\left(\mathbf{G}^{\mathrm{T}} \mathbf{G}\right)^{-1} \mathbf{G}^{\mathrm{T}} \mathbf{b}-\mathbf{b}
$$

due to

$$
\operatorname{det}\left(\mathbf{G}^{\mathrm{T}} \mathbf{G}\right)=\operatorname{det}\left(\mathbf{G}^{\mathbf{T}}\right) \operatorname{det}(\mathbf{G}) .
$$

According to the topic, the positions of the sources $A P 1, A P 2$, and $A P 3$ are independent of each other, so the vector group formed $\mathbf{G}$ is linearly independent, and it is obvious that

$$
\operatorname{det}\left(G^{T}\right) \neq 0 \text { and } \operatorname{det}(G) \neq 0 \Longrightarrow \operatorname{det}\left(G^{T} G\right) \neq 0 .
$$

Therefore, the term $\left(\mathbf{G}^{\mathrm{T}} \mathbf{G}\right)^{-1}$ on the right side of equation (36) must exist. Make

$$
c=\left\|\mathbf{m}_{\varepsilon}\right\| .
$$

Substituting formula (32) finally obtains

$$
\rho=1-\frac{\mathbf{m}_{\varepsilon}}{\max _{i} \mathbf{m}_{\varepsilon, i}}, \quad i=1,2, \ldots, m n k,
$$

where $\mathbf{m}_{\varepsilon, i}$ is the error vector of $\mathbf{m}_{\varepsilon}$ corresponding to the $i$-th equation group. For the mnk combinations determined by equation (29), $\rho$ is sorted according to equations (36) and (39) and sorted, the equation group with the largest $\rho$ value is the best choice, and then the $\mathbf{W}$ calculated according to equation (21) is the pending optimal solution for the measuring point $p(x, y)$.

\section{Experiment}

The experimental environment selects an office area on the third floor of an office building. The office area is an approximately rectangular area with a length and width of approximately 50 meters $\times 25$ meters, as shown in Figure 4 .

The upper left corner of the figure is used as the origin of the coordinates, the horizontal direction to the right is the horizontal axis, and the vertical direction is the vertical axis to establish the coordinate system. For comparison, the experiment was divided into two groups. The first group of experiments uses the traditional three-sided positioning method for testing. The entire indoor environment is regarded as a whole. In the test environment, 50 test points are randomly selected for position calibration as shown in Figure 5.

Then, further estimate and calibrate the values of the transmission model parameters $A$ and $n$ expressed by equation (3). Then, select 30 random positions for positioning test and record the actual coordinates and positioning results of the random positions. In the second set of experiments, the improved method proposed in this paper is used to carry out regional preprocessing of indoor space, in turn constructing the area set, parameter set, and decision domain set corresponding to each plane, and applying the regional confidence method to the distance measurement and positioning in the positioning stage. The solution results are constrained and filtered, and the positioning results are recorded. In order to reduce the interference factors, the two sets of experiments used the same set of test points in the

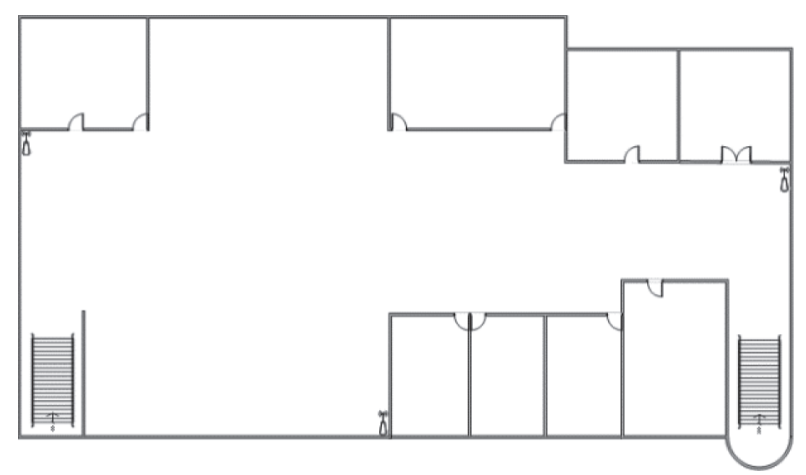

Figure 4: Indoor layout of the experimental environment.

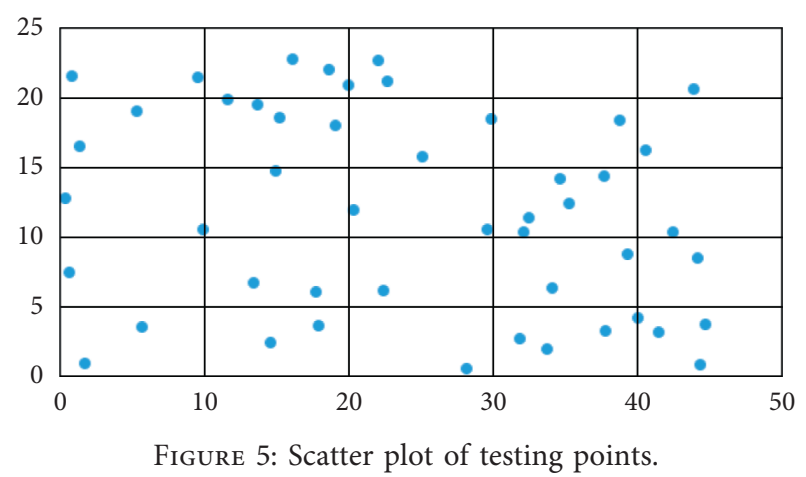

estimation and calibration of the model parameters. For the same reason, the positioning test point data of the two sets of experiments are also the same.

Suppose the actual position of the point to be measured is $W_{0}$, the positioning result solved by the traditional threesided positioning method in the first group of experiments is $W_{1}$, and the positioning result solved by the improved method in the second group of experiments is $W_{2}$, $e_{i}=W_{i}-W_{0}, i=1,2$. That is, $e_{1}$ is the positioning error of the traditional three-sided positioning method obtained by experiments, and $e_{2}$ is the positioning error of the improved method. Process the test data of the two sets of experiments according to the above formula, calculate and plot the positioning error corresponding to each test point, and set the horizontal axis as the test point number and the vertical axis as the positioning error, as shown in Figure 6.

It can be seen from the figure that the positioning error of the traditional three-sided positioning method is relatively large, the positioning error of individual test points even exceeds $10 \mathrm{~m}$, and the fluctuation of the error is relatively large, which indicates that the positioning error of the traditional three-sided positioning method is relatively discrete. The positioning error range using the improved method in this paper is basically controlled within about $2 \mathrm{~m}$.

Set the horizontal axis as the positioning error and the vertical axis as the cumulative error probability. Plot the distribution diagram of cumulative error probability accordingly, as shown in Figure 7. $F_{1}$ and $F_{2}$ are the distribution of cumulative error probability of the first and second sets of experimental data, respectively. It can be clearly seen 


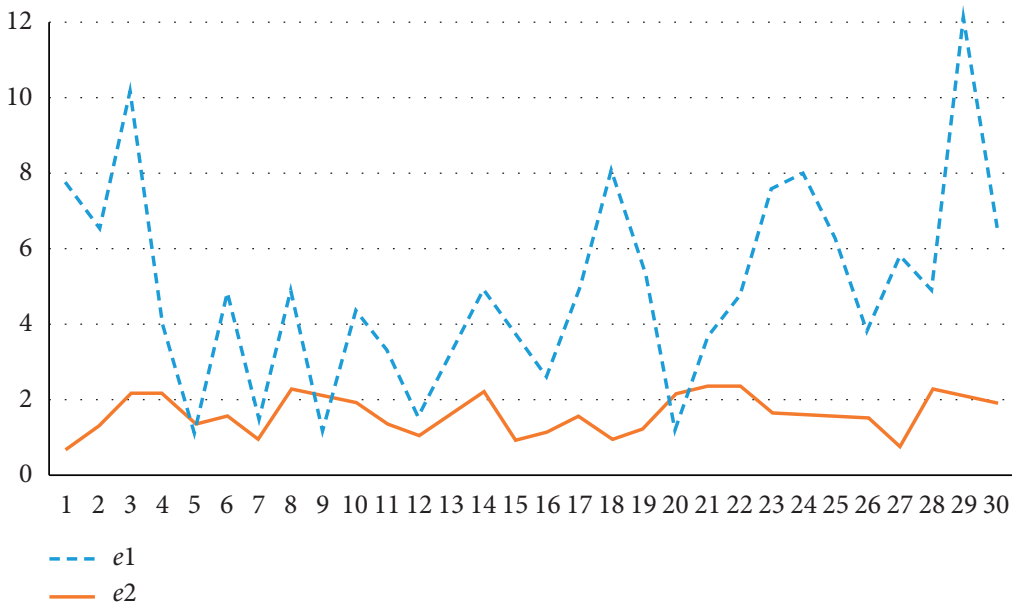

FIgURE 6: Comparison on positioning errors.

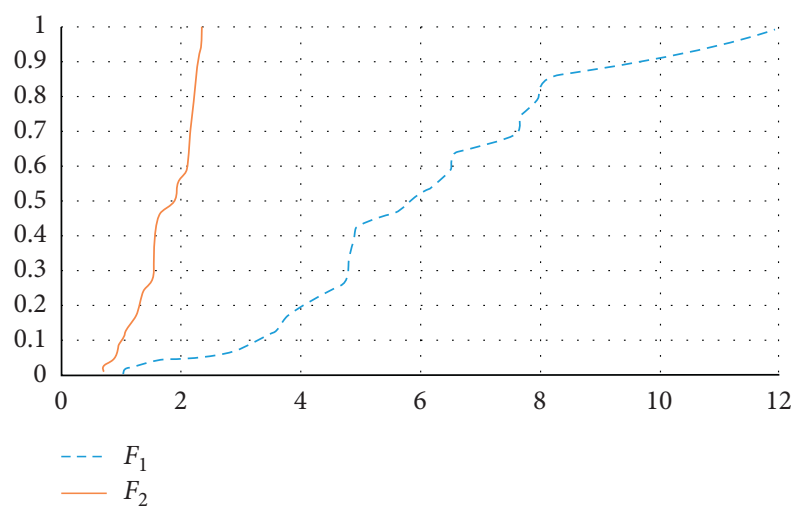

FIGURE 7: Distribution of cumulative error probability.

from the figure that the distribution curve of cumulative error probability of the second group that used the improved positioning method reaches the peak soon after the horizontal axis crosses $2 \mathrm{~m}$, which indicates that the positioning error is basically distributed within $2.2 \mathrm{~m}$, while the distribution curve of cumulative error probability in the first group which used traditional positioning is relatively smooth, and as the distance increases, the error convergence is slower. Relatively speaking, the positioning effect of the second group that used the improved method is obviously better.

\section{Conclusion and Future Work}

The propagation of wireless signals in an indoor environment is a fairly complicated process. The RSS value of a wireless signal at a specific indoor location is restricted by many factors. The traditional indoor positioning technique based on RSS considers the indoor environment as a unified whole. Since it ignores the differences in the wireless signal transmission paths of various points in the indoor environment, the positioning effect is not satisfactory. The method proposed in this paper takes sufficient account of these differences, and it takes advantage of the prior knowledge of the layout algorithm of the known indoor environment, by the way of the localization of the indoor environment and the approaching positioning to solve this problem. It is worth noting that in the application, the first problem needs to be solved is the acquisition of indoor environment data of the building in the training phase. In the current field of building and construction, the building information model (BIM) has become popular, and many buildings are carried on by BIM in the design phase. Therefore, the BIM can be used to obtain data on the indoor environment, saving a lot of complicated work in the early phase. In addition, in the indoor environment, due to the unpredictable shielding effect of indoor pedestrians on the wireless signal transmission path and the multipath effect caused by the reflection of the wireless signal by the wall plate in the building, these factors will reduce the positioning accuracy. Therefore, adding Gaussian filtering and Kalman filtering to the RSS data acquisition module of the RSS indoor positioning equipment can weaken this effect to a certain extent, and this follow-up work needs to be further developed in the future.

\section{Data Availability}

The data are available on requesting the corresponding author Xingsi Xue, whose e-mail is jack8375@gmail.com.

\section{Conflicts of Interest}

The authors declare that there are no conflicts of interest regarding the publication of this paper.

\section{Acknowledgments}

This work was supported by the Guangxi Key Laboratory of Automatic Detecting Technology and Instruments (no. YQ20206), the Program for New Century Excellent Talents in Fujian Province University (no. GY-Z18155), the Scientific Research Foundation of Fujian University of Technology (no. GY-Z17162), the Science and Technology Planning Project in Fuzhou City (no. 2019-G-40), and the 
Foreign Cooperation Project in Fujian Province (no. 2019I0019).

\section{References}

[1] Xi Rui, Y. Li, and M. Hou, "Summary of indoor positioning methods," Computer Science, vol. 43, no. 4, pp. 1-32, 2016.

[2] Y. Zhang, X. Xu, and $\mathrm{K} . \mathrm{Xu}$, "WLAN indoor positioning system based on weighted centroid method," Journal of Electronic Measurement and Instrument, vol. 29, no. 7, pp. 1036-1041, 2015.

[3] R. Zhao, B. Zhong, Z. Zhu et al., "Overview of indoor positioning technique and applications," Electronic Technology, vol. 27, no. 3, pp. 154-157, 2014.

[4] X. Shi, A. Yin, and X. Chen, "Multi-dimensional indoor positioning algorithm based on RSSI," Journal of Scientific Instrument, vol. 35, no. 2, pp. 261-268, 2014.

[5] L. Pei, D. Liu, and J. Qian, "Overview of indoor positioning technique and applications," Navigation Positioning and Timing, vol. 4, no. 3, pp. 1-10, 2017.

[6] X. Xue and Y. Wang, "Optimizing ontology alignments through a memetic algorithm using both MatchFmeasure and unanimous improvement ratio," Artificial Intelligence, vol. 223, pp. 65-81, 2015.

[7] X. Xue and Y. Wang, "Using memetic algorithm for instance coreference resolution," in Proceedings of the IEEE Transactions on Knowledge and Data Engineering, Helsinki, Finland, May 2016.

[8] X. Xue and J. Lu, "A compact brain storm algorithm for matching ontologies," IEEE Access, vol. 8, pp. 43898-43907, 2020.

[9] X. Xue, "A compact firefly algorithm for matching biomedical ontologies," Knowledge and Information Systems, vol. 62, no. 7, pp. 2855-2871, 2020.

[10] X. Xue, X. Wu, and J. Chen, “Optimizing biomedical ontology alignment through a compact multiobjective particle swarm optimization algorithm driven by knee solution," Discrete Dynamics in Nature and Society, vol. 2020, Article ID 4716286, 10 pages, 2020.

[11] X. Xue and J. Chen, "Using compact evolutionary tabu search algorithm for matching sensor ontologies," Swarm and Evolutionary Computation, vol. 48, pp. 25-30, 2019.

[12] P. Hu, J.-S. Pan, and S.-C. Chu, "Improved binary grey wolf optimizer and its application for feature selection," Knowledge-Based Systems, vol. 195, pp. 1-14, 2020.

[13] C.-H. Chen, "A cell probe-based method for vehicle speed estimation," IEICE Transactions on Fundamentals of Electronics, Communications and Computer Sciences, vol. E103.AA, no. 1, pp. 265-267, 2020.

[14] C.-H. Chen, F. Song, F.-J. Hwang et al., "A probability density function generator based on neural networks," Physica A: Statistical Mechanics and Its Applications, vol. 541, pp. 1-10, 2020.

[15] C.-H. Chen, F.-J. Hwang, and H.-Y. Kung, "Travel time prediction system based on data clustering for waste collection vehicles," IEICE Transactions on Information and Systems, vol. E102.D, no. 7, pp. 1374-1383, 2019.

[16] C. Miao, Research on WLAN Indoor Positioning and Tracking System Based on Signal Strength, Wuhan University, Wuhan, China, 2012.

[17] Z. Jin, W. Li, J. Liang et al., "Design of indoor positioning system based on KNN-SVM algorithm," Journal of Huazhong University of Science and Technology (Natural Science Edition), vol. 43, no. S1, pp. 517-520, 2015.
[18] G. Shi, B. Wang, and B. Wu, "A summary of indoor positioning methods based on WiFi and mobile intelligent terminals," Computer Engineering, vol. 41, no. 9, pp. 39-44+50, 2015.

[19] K. Shi, H. Chen, and R. Zhang, "An 802.11 wireless indoor positioning method based on support vector regression," Journal of Software, vol. 25, no. 11, pp. 2636-2651, 2014.

[20] S. Cao, "Research progress of indoor positioning technique and system," Computer System Application, vol. 22, no. 9, pp. 1-5, 2013.

[21] Z. Deng, Research on WLAN Indoor Positioning Technique Based on Learning Algorithm, Harbin Institute of Technology, Harbin, China, 2012. 